\title{
An Effector of Hemoglobin Structure: The Guanosine 3', 5'-Triphosphate
}

\author{
Saeed Rezaei-Zarchi, ${ }^{a}$ Saber Imani, ${ }^{\text {b }}$ Safieh Soufian, ${ }^{\mathrm{c}}$ Yunes Panahi, ${ }^{\mathrm{b}}$ \\ Ali Akbar Saboury, ${ }^{\mathrm{d}}$ and Mohammad Reza Arefie, ${ }^{\mathrm{e}}$ \\ ${ }^{a}$ Department of Biology, Payame Noor University, Yazd, Iran \\ ${ }^{\mathrm{b}}$ Chemical Injuries Research Center, Baqiyatallah University of Medical Sciences, Tehran, Iran \\ ${ }^{\mathrm{c}}$ Department of Biology, Payame Noor University, Arak, Iran \\ ${ }^{\mathrm{d}}$ Institutes of Biochemistry and Biophysics, University of Tehran, Tehran, Iran \\ ${ }^{\mathrm{e}}$ Department of Civil Engineering and Nano Research Center, Taft branch, Islamic Azad University, Taft, Iran
}

RECEIVED JULY 22, 2011; REVISED OCTOBER 30, 2011; ACCEPTED NOVEMBER 4, 2011

\begin{abstract}
The effect of guanosine 3', 5'-triphosphate (GTP) on the hemoglobin structure was studied by UV-visible, fluorescence and circular dichroism (CD) spectroscopies, and cyclic voltammetry. UV-visible absorption spectra showed an increase in absorbance in the regions of $420 \mathrm{~nm}$ and $280 \mathrm{~nm}$. Fluorescence spectra showed that the Trp fluorescence intensity increased upon excitation at $280 \mathrm{~nm}$, when guanosine 3', 5'-triphosphate concentration was increased in hemoglobin solution. Along with the increased fluorescence intensity, a slightly shift of $\lambda_{\max }$ was also observed toward the higher wavelengths. CD spectral analysis demonstrated a significant decrease in negative ellipsity in the region of 205-235 $\mathrm{nm}$. After adding guanosine 3', 5'-triphosphate to the hemoglobin solution $\alpha$-helix structure decreases by $20 \%$ while $\beta$-sheet conformation increases by $9 \%$. The effects of GTP on hemoglobin resulted in a $61 \mathrm{mV}$ shift in the cathodic and $40 \mathrm{mV}$ for anodic peak of hemoglobin in the CD. Our data showed the change of secondary and tertiary structure of hemoglobin in the presence of guanosine 3', 5'-triphosphate. (doi: $10.5562 /$ cca1955)
\end{abstract}

Keywords: hemoglobin structure, guanosine 3', 5'-triphosphate

\section{INTRODUCTION}

Hemoglobin is a hetero-tetramer, consisting of two $\alpha$-subunits and two $\beta$-subunits (a "dimer of dimers" $[\beta(2): \alpha(2)])$. Each polypeptide chain contains a prosthetic heme group, which cooperatively binds and releases oxygen. ${ }^{1-3}$ Hemoglobin undergoes major tertiary and quaternary conformational changes as it equilibrates between a low-affinity deoxy T-state and a high-affinity oxy R-state. ${ }^{4}$ Organic phosphates and some heterotrophic effectors, such as protons, anions and carbon dioxide, although bound spatially at the remote sites, could affect the oxygenation process and have been shown to affect the oxidation process as well. ${ }^{1,5-7}$ Some organic phosphates, such as diphosphoglycerate (DPG), bind with a site of hemoglobin, in 1:1 stoichimetry, which is located at the entrance of the cavity between two $\beta$-subunits. These subunits contribute positively charged residues to complement the negatively charged DPG phosphate groups, namely Val1, His2 and Lys82 of both the $\beta$-chains.
Moreover, inositol hexaphosphate (IHP) and DPG can bind to the hemoglobin at the same site. ${ }^{8}$ The allosteric function of these materials leads to its binding with the hemoglobin and changing the R-like state of hemoglobin to T-like state, and decreasing the oxygen affinity of hemoglobin. ${ }^{1,8}$ It is known that GTP is very important factor in the erythroid differentiation and stimulates it for the synthesis of hemoglobin and other proteins. ${ }^{9}$ GTP has the main role in activating or deactivating some proteins in the cell, such as kinases and G-proteins. ${ }^{10}$ Since, its important roles in erythroid differenciation, where hemoglobin is generated, investigations about the effects of this material on the structural changes of hemoglobin and its affinity to oxygen are very important. Tamburrini et al. have reported that the affinity of hemoglobin, for oxygen, was reduced dramatically in the present of GTP. ${ }^{11}$ However, there are still unanswered questions like: how GTP affects the hemoglobin structure?

Here we report the investigation of the structural changes of hemoglobin, related to the binding of GTP,

\footnotetext{
* Author to whom correspondence should be addressed. (E-mail: arefi@taftiau.ac.ir)
} 
by UV-visible, fluorescence and CD spectroscopies, and cyclic voltammetry.

\section{EXPERIMENTAL}

\section{Materials}

Human adult hemoglobin ( $\mathrm{HbA}), \mathrm{KNO}_{3}, \mathrm{GTP}$, 3-( $N$-morpholino)propanesulfonic acid (MOPS) (99\%) and KI were purchased from Sigma. Double distilled water was used in all the experiments. Stock solutions were stored at $4{ }^{\circ} \mathrm{C}$.

\section{UV-Visible Spectroscopy}

The UV-Visible absorption spectra were measured with a UV60A spectrophotometer (Shimadzu, Japan), using a $1 \mathrm{~cm}$ quartz cuvette. Spectral changes of $1.5 \mu \mathrm{M}$ hemoglobin were monitored after adding different concentration of GTP by recording the UV-visible absorption (250-500 nm), (0.1 M MOPS buffer; $\mathrm{pH} 7.0$ ).

\section{Fluorescence Experiments}

A fluorescence spectrophotometer (Model MPF-4, Hitachi, Japan), equipped with a temperature controller bath (Model NTB-211), was used for all of the fluorescence experiments. The excitation wavelength, adjusted at 280 $\mathrm{nm}$ during the whole experiment, was specific for the excitation of the tryptophan residues in the protein structure. The emission spectra were recorded between the wavelengths of 300 to $400 \mathrm{~nm}$ at the band-width of $10 \mathrm{~nm}$. Hemoglobin sample was provided at the concentration of $1.5 \mu \mathrm{M}$ in a $0.1 \mathrm{M}$ MOPS buffer $(\mathrm{pH} 7.0)$.

\section{Spectroscopy}

CD spectra were collected, between 190 and $260 \mathrm{~nm}$, with an Aviv CD spectrometer at $25^{\circ} \mathrm{C}$. The $\mathrm{CD}$ assay was performed using a $1 \mathrm{~mm}$ path length cell with the protein concentration of $0.2 \mu \mathrm{M}$ in the $0.1 \mathrm{M}$ MOPS buffer ( $\mathrm{pH}$ 7.0) with different concentration of GTP. The molar elasticity values were collected from the CD spectra. CDNN program was used to calculate the percentages of $\alpha$-helix and $\beta$-sheets of all the samples used. $^{12}$

\section{Electrochemical Experiments}

Electrochemical measurements were carried out with a Potentiostat/Galvanostat (Model 263A, EG\&G, USA) using a single-compartment voltammetric cell, equipped with a platinum rod auxiliary electrode, an $\mathrm{Ag} / \mathrm{AgCl}$ reference electrode (Metrohm) and an iodide modified silver working electrode with a disk diameter of $1 \mathrm{~mm}$ (Azar Electrode Co; Iran). Iodide modified working electrode was prepared by the method stated by some previous workers. ${ }^{13-15}$ First, the silver electrode was mechanically polished twice with alumina (particle sizes 10 and $0.06 \mu \mathrm{m}$, respectively) to a mirror finish. Then, the adsorbed species were further removed by etching the electrode in $10 \% \mathrm{HNO}_{3}$ solution for 1 minute. Finally, the electrode was thoroughly washed with double distilled water followed by treating it in an ultrasonic bath for about 3 minutes. After this pre-treatment, the substrate silver electrode was immerse in a $0.1 \mathrm{~mol} / \mathrm{L} \mathrm{KI}$ solution for 5 minutes and then thoroughly rinsed with double distilled water to remove any physic-absorbed material. The iodide modified silver electrode was then ready for use. All the solutions were de-aerated by bubbling with high purity nitrogen for at least 30 minutes before the experiments.

\section{RESULTS AND DISCUSSION}

UV-visible absorption spectra were recorded for the conformational changes of hemoglobin $(1.5 \mu \mathrm{M})$, in the solution, when GTP was added at different concentrations. As shown in Figure 1, an increase in absorbance was seen in the region of $420 \mathrm{~nm}$ (due to the Soret absorption by the heme system) and $280 \mathrm{~nm}$ (due to tryptophan absorption). ${ }^{16,17}$ Naturally, the tryptophan residue (hydrophobic amino acid) of hemoglobin is confined inside the protein molecule. ${ }^{16}$ Increase of absorption in the region of $280 \mathrm{~nm}$ shows that interaction of GTP, with hemoglobin, caused the tryptophan residues to migrate towards the protein surface and increased surface accessibility of tryptophan. ${ }^{16-18}$ While, the increased absorption at $420 \mathrm{~nm}$ can be because of the same effect of GTP on the surface accessibility of heme group, as a result of conformational changes in hemoglobin structure during the interaction of GTP with hemoglobin. ${ }^{16-18}$ In fact, the interaction between protein and GTP caused the changes in protein structure and increased the absorption of Trp residues/heme groups.

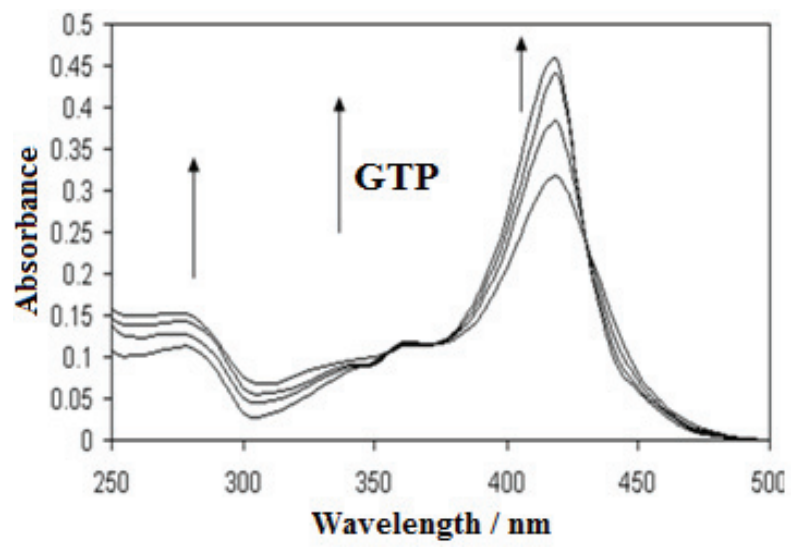

Figure 1. The effect of GTP concentration (0, 75, 150, 225 $\mu \mathrm{M})$ on $\mathrm{UV}$-visible spectrum of hemoglobin $(1.5 \mu \mathrm{M})$. 


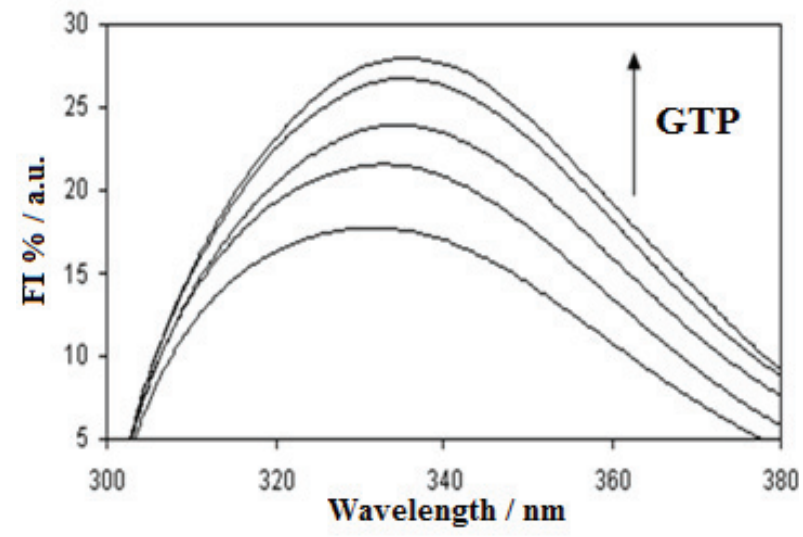

Figure 2. The effect of GTP concentration $(0,75,150,225$, $300 \mu \mathrm{M})$ on florescence spectrum of hemoglobin $(1.5 \mathrm{M})$. $\left(\lambda_{\max }=280 \mathrm{~nm}\right)$.

Figure 2 shows the gradual increase of the intrinsic tryptophan fluorescence during the addition of GTP in the solution containing $1.5 \mu \mathrm{M}$ hemoglobin. Excited at $280 \mathrm{~nm}$, the Trp intensity increased with increase in GTP concentration. Along with the increased fluorescence intensity, a slightly shift of $\lambda_{\max }$ was also observed toward the higher wavelengths. So, our data showed a close correlation between the intrinsic fluorescence intensity of tryptophan and the results obtained by the UV-visible spectrophotometry. With the increase of GTP concentration to hemoglobin solution, an increase of fluorescence emission was observed, which can also be the outcome of more contact of tryptophan residues to the environment. ${ }^{17-20}$ In general, a Trp in a hydrophobic environment exhibits maximum emission at lower wavelengths as compared to a hydrophilic- or exposed-environment. ${ }^{17-20}$ As it is shown in Figure 2, with the increase of GTP as well as the Trp fluorescence intensity, $\lambda_{\max }$ was slightly shifted toward the higher wavelengths (red shift). This observation shows that when the protein interacted with the GTP, Trp residues were exposed to more hydrophilic environment, which also shows the structural change and unfolding of the protein.

Figure 3 shows GTP-induced conformational changes of hemoglobin by $\mathrm{CD}$ spectral analysis. The CD spectra, at far UV region of GTP-hemoglobin, are very different from the spectrum of hemoglobin. A significant decrease in negative ellipsity was seen in the region of 205-235 nm, which shows a significant change in the secondary structure of hemoglobin during its interaction with GTP. ${ }^{16-20}$ After adding $30 \mu \mathrm{M}$ GTP to $0.2 \mu \mathrm{M}$ hemoglobin solution, the $\alpha$-helix structure of the protein decreased approximately $20 \%$, while $\beta$-sheet conformation increased approximately $9 \%$. These results show some changes in the secondary structure of hemoglobin after the interaction with GTP.

Figure 4 shows the cyclic voltammetric effects of $\mathrm{GTP}$, on the hemoglobin $(30 \mu \mathrm{M})$ in solution, by using

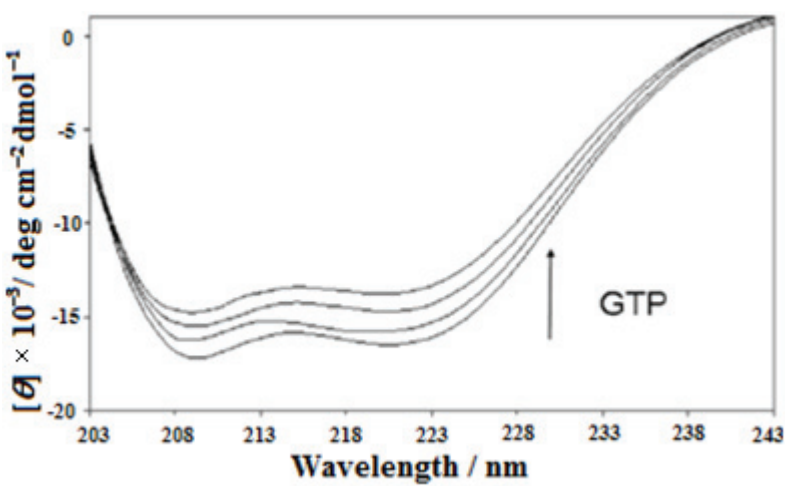

Figure 3. The effect of GTP concentration $(0,10,20,30 \mu \mathrm{M})$ on $\mathrm{CD}$ spectrum of hemoglobin $(0.2 \mu \mathrm{M})$.

iodide-modified silver electrode. Inset of Figure 4 shows the relationship between $E^{\circ 1}$ and [GTP]. The cathodic peak of hemoglobin shifts $61 \mathrm{mV}$ and anodic peak shifts $40 \mathrm{mV}$ to positive direction with the GTP concentration ranging from 0 to $4.5 \mathrm{mM}$. It almost makes no cyclic voltammogram (CV) changes for hemoglobin at the GTP concentrations above $4.5 \mathrm{mM}$. CV studies also show no shift in both the cathodic and anodic peaks in the presence of $4 \mathrm{mM}$ guanosine (data not shown). These results indicate that only the phosphate part of GTP can interact with hemoglobin. Positive shift of cathodic and anodic peaks of hemoglobin by GTP interaction means the easier redox behavior of hemoglobin in the presence GTP. ${ }^{21}$ It seems that GTP can change the structure and cause the unfolding of hemoglobin. While, the heme group would change its position from the inside to the surface of hemoglobin. ${ }^{21-23}$ It seems that GTP can bind and change the secondary and tertiary structures of hemoglobin. We showed that reason of the decreased affinity of hemoglobin for oxygen, as stated by Tamburrini et al., ${ }^{11}$ may be because of the changed secondary and tertiary structures of hemoglobin. Our data suggest that GTP acts as a regulator of hemoglobin's affinity for oxygen by structural changes in the protein.

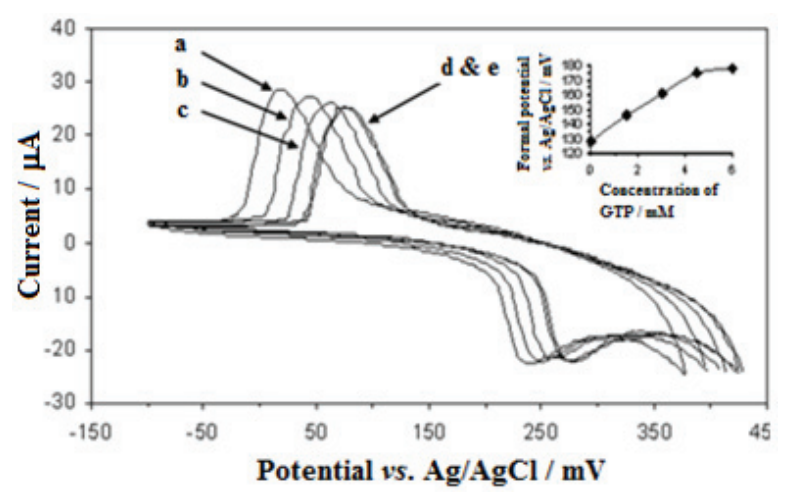

Figure 4. Cyclic voltammograms of hemoglobin $(30 \mu \mathrm{M})$ in the presence of a) 0, b) 1.5 , c) 3.0 , d) 4.5 and e) $6.0 \mathrm{mM}$ of GTP, respectively. Inset shows the plot of [GTP] vs. $E^{\circ}$. 


\section{CONCLUSION}

Thus, we showed that GTP interacts with hemoglobin and changes the secondary and tertiary structures of hemoglobin. Trp residues were exposed to more hydrophilic environment, which is accompanied with an increase in the $\beta$-structure and a decrease in the $\alpha$-helix structure of the protein. So, GTP can affect hemoglobin as a ligand to regulate its affinity for oxygen.

Acknowledgements. The financial supports of Payame Noor University, Yazd, Iran for the present project are gratefully acknowledged.

\section{REFERENCES}

1. M. Pomponi, E. Gavuzzo, C. Bertonati, A. E. Derocher, C. Lydersen, Y. Wiig, and K. M. Kovacs, Biochimie 86 (2004) 927-932.

2. D. Ajloo, A. A. Moosavi-Movahedi, M. Sadeghi, and H. Gharibi, Acta Biochim. Pol. 49 (2002) 459-470.

3. J. Baldwin and C. Chothia, J. Mol. Biol. 129 (1979) 175-220.

4. A. Tsuneshige, S. Park, and T. Yonetani, Biophys. Chem. 98 (2002) 49-63.

5. M. Marta, M. Patamia, A. Colella, S. Sacchi, M. Pomponi, and K. Kovacs, Biochem. 37 (1998) 14024-14029.

6. K. M. Faulkner, A. L. Crumbliss, and C. Bonaventura, J. Biol. Chem. 270 (1995) 13604-13612.

7. C. Poyart, M. C. Marden, and J. Kister, Methods Enzymol. 232 (1994) 496-513.

8. M. Laberge, I. Kovesi, T. Yonetani, and J. Fidy, FEBS Lett. 579
(2005) 627-632.

9. F. Osti, F. G. Corradini, S. A. Hanau, M. Matteuzzi, and B. Gambari, Haematologica 82 (1997) 395-401.

10. M. A. Rubin, A. C. Medeiros, P. C. B. Rocha, C. Livi, B. Ramirez, and G. D. O. Souza, Neurochem. Res. 22 (1997) 181187.

11. M. Tamburrini, C. Verde, A. Olianas, B. Giardina, M. Corda, and M. Sanna, J. Biochem. 268 (2001) 4104-4111.

12. G. B. Butler, K. F. O'Driscoll, and G. L. Wilkes, Macromol. Chem. Phys. 34 (1994) 325-373.

13. C. Li. G. Fan, Y. Zhuang, J. Zhu, and D. Zhu, Electroanal. 12 (2000) 205-208.

14. M. S. Sibbald, G. Chumanov, and T. M. Cotton, J. Electroanal. Chem. 438 (1997) 179-185.

15. M. S. Sibbald, G. Chumanov, and T. M. Cotton, J. Phys. Chem. 100 (1996) 4672.

16. M. R. Dayer, A. A. Moosavi-Movahedi, P. Norouzi, H. Ghourchian, and S. Safarian, J. Biochem. Mol. Biol. 35 (2002) 364-370.

17. A. A. Moosavi-Movahedi, K. Nazari, and A. A. Saboury, Biointerfaces J. 9 (1994) 3-4.

18. D. Ajloo, A. A. Moosavi-Movahedi, M. Sadeghi, and H. Gharibi, Acta Biochim. Pol. 49 (2002) 459-470.

19. Y. Sun, X. Liu, C. Fan, W. Zhang, and G. Li, Bioelectrochem. J. 64 (2004) 23-27.

20. H. Mizukoshi, M. Itoh, S. Matsakawa, K. Mawatari, and Y. Yoneyama, Biophys. Acta 700 (1982) 143-147.

21. W. Peng, X. Liu, W. Zhang, and G. Li, Biophys. Chem. 106 (2003) 267-273.

22. K. M. Faulkner, C. Bonaventura, and A. L. Crumbliss, Chim. Acta 226 (1994) 187-194.

23. Q. C. Li and P. A. Mabrouk. J. Biol. Inorg. Chem. 8 (2003) 83-94. 